6. E. P. Lane, $A$ treatise on projective differential geometry, The University of Chicago Press, 1942.

7. - - The correspondence between the tangent plane of a surface and its point of contact, Amer. J. Math. vol. 48 (1926).

8. E. J. Wilczynski, Projective differential geometry of curved surfaces, Second memoir, Trans. Amer. Math. Soc. vol. 9 (1908).

Michigan State College

\title{
TWO NOTES ON NILPOTENT GROUPS
}

\author{
R. C. LYNDON
}

I

We extend a theorem of Rédei and Szép. ${ }^{1}$ Our proof is quite straightforward, and employs a method of considerably more general applicability. ${ }^{2}$

The lower central series of a group $G$ is formed by taking $G_{1}=G$, and successively defining $G_{n+1}$ to be the commutator $\left(G_{n}, G\right)$. $G$ is nilpotent if some $G_{N+1}=1$. If $A$ and $B$ are subgroups of $G, A \vee B$ is the subgroup generated by the elements of $A$ and of $B$ together, and $A^{m}$ the subgroup generated by the $m$ th powers of elements of $A$.

TheOREM. Let $A$ and $K$ be subgroups of a nilpotent group $G$, and let $A^{m^{\circ}}=1$ for some integer $m^{e}$. Then, for any $n \geqq 1$,

$$
(A \vee K)_{n}=\left(A^{m} \bigvee K\right)_{n} \text { implies }(A \vee K)_{n}=K_{n}
$$

We may clearly suppose that $G=A \vee K$. The elements of $G_{r}$ can be written as products of commutators of order $r$ :

$$
\left(x_{1}, \cdots, x_{r}\right)=\left(\left(\cdots\left(\left(x_{1}, x_{2}\right), x_{3}\right) \cdots, x_{r-1}\right), x_{r}\right) .
$$

Let $C_{r}$ be the subgroup generated by those commutators for which

Received by the editors January 7, 1952.

${ }^{1}$ L. Rédei and J. Szép, Monatshefte für Mathematik vol. 55, p. 200. The present proof avoids "counting arguments" and the attendant finiteness conditions; for $n=1$ the present argument reduces substantially to that of Rédei and Szép. We remark that the hypothesis $A^{m^{e}}=1$ admits various modifications.

2 The basic idea of "expanding" words in commutators of ascending order has been exploited by P. Hall, Proc. London Math. Soc. vol. 36, p. 29; and by O. Grün, J. Reine Angew. Math. vol. 182, p. 158. See also W. Magnus, Monatschefte für Mathematik vol. 47, p. 307, and K. T. Chen, Proceedings of the American Mathematical Society vol. 3, p. 44. 
some $x_{i}$ is in $A$, and $D_{r}$ by those for which some $x_{i}$ is in $A^{m}$. From the identity

$$
(x, y z)=(x, y)(x, z)(z, x, y)
$$

it follows that all commutators are linear in the $x_{i}$, modulo commutators of higher order. In particular, it follows that

$$
\begin{gathered}
(A \vee K)_{n}=K_{n} \vee C_{n}, \\
\left(A^{m} \vee K\right)_{n}=K_{n} \vee D_{n,} \\
D_{n} \subset C_{n}^{m} \vee C_{n+1},
\end{gathered}
$$

and, since $A^{m^{e}}=1$, that

$$
C_{n}^{m^{6}} \subset C_{n+1}
$$

From the hypothesis that $(A \bigvee K)_{n}=\left(A^{m} \bigvee K\right)_{n}$, hence that $K_{n} \bigvee C_{n}$ $=K_{n} \bigvee D_{n}$, we have $C_{n} \subset K_{n} \bigvee D_{n}$ and, from (1),

$$
C_{n} \subset K_{n} \vee C_{n}^{m} \vee C_{n+1}
$$

By the evident rule $(L \bigvee M)^{m} \subset L^{m} \bigvee M^{m} \bigvee(L, M)$, from

$$
C_{n} \subset K_{n} \vee C_{n}^{r} \vee C_{n+1}
$$

we deduce that

$$
\begin{aligned}
& C_{n}^{m} \subset K_{n}^{m} \vee C_{n}^{r m} \bigvee C_{n+1}^{m} \vee G_{2 n}, \\
& C_{n}^{m} \subset K_{n} \vee C_{n}^{r m} \vee C_{n+1},
\end{aligned}
$$

and, by (3), that

$$
C_{n} \subset K_{n} \vee C_{n}^{r m} \vee C_{n+1}
$$

Applying this argument $e-1$ times to (3) gives

$$
C_{n} \subset K_{n} \vee C_{n}^{m^{e}} \vee C_{n+1}
$$

whence, by (2),

$$
C_{n} \subset K_{n} \vee C_{n+1}
$$

From the Lie-Jacobi congruences

$$
(x, y)(y, x)=1, \quad(x, y, z)(y, z, x)(z, x, y) \equiv 1\left(\bmod G_{4}\right),
$$

it follows that every $\left(x_{1}, \cdots, x_{k+1}\right)$ with $x_{k+1}$ in $A$ is expressible, modulo $G_{k+2}$, as a product of such factors with $x_{i}$ in $A$ for some $i \leqq k:$ in short, 


$$
C_{k+1} \subset\left(C_{k}, G\right) \vee C_{k+2}=\left(C_{k}, K\right) \vee\left(C_{k}, A\right) \vee C_{k+2}
$$

Assuming now

$$
C_{k} \subset K_{k} \vee C_{k+1}
$$

and substituting, we obtain

$$
\begin{aligned}
& \left(C_{k}, K\right) \subset K_{k+1} \vee C_{k+2}, \\
& \left(C_{k}, A\right) \subset\left(K_{k}, A\right) \vee C_{k+2} \subset\left(C_{k}, K\right) \vee C_{k+2} \subset\left(C_{k}, K\right),
\end{aligned}
$$

whence

$$
C_{k+1} \subset K_{k+1} \bigvee C_{k+2}
$$

By iteration, it follows from (4) that

$$
C_{n} \subset K_{n} \vee K_{n+1} \vee \cdots \vee K_{N} \vee C_{N+1} \subset K_{n} \vee C_{N+1}
$$

Since $G_{N+1}=1$ by hypothesis,

$$
C_{n} \subset K_{n}
$$

whence $K_{n} \vee C_{n}=K_{n}$ and $(A \bigvee K)_{n}=K_{n}$, as required.

\section{II}

By a uniform method ${ }^{3}$ we establish easily two results that are fairly obvious from well known considerations, and a further result (Theorem 2.1) which answers for nilpotent groups a question regarding identical relations in groups that was raised by B. H. Neumann. ${ }^{4}$

We employ standard notation for commutators: $\left(x_{1}, \cdots, x_{n}\right)$ $=\left(\cdots\left(\left(x_{1}, x_{2}\right), x_{3}\right), \cdots, x_{n}\right)$, and for the lower central series: $G=G_{1}, G_{n+1}=\left(G_{n}, G\right)$.

LEммA 1. Let $F$ be a finitely generated free group, and $R$ a normal subgroup of $F$. Then, for each $n \geqq 1, R=\left[S_{n}, R_{n+1}\right]$, the normal subgroup generated by a finite set $S_{n}$ together with $R_{n+1}=R \cap F_{n+1}$.

Proof. Proceed inductively from the vacuous case $n=0$. Since $F_{n+1} / F_{n+2}$ is a finitely generated abelian group, so is its subgroup $R_{n+1} / R_{n+2}$. Let $T=\left\{r_{i}\right\}$ be a finite set of elements of $R_{n+1}$ such that the cosets $r_{i} R_{n+2}$ generate $R_{n+1} / R_{n+2}$. Evidently $R=\left[S_{n}, R_{n+1}\right] \mathrm{im}$ plies $R=\left[S_{n}, T, R_{n+2}\right]$.

THEOREM 1.1. Every finitely generated nilpotent group is definable by a finite set of relations.

3 For the method, see references given in footnote 2 .

B. H. Neumann, Math. Ann. vol. 114, p. 506. Theorem 2.1 was announced by the author in Bull. Amer. Math. Soc. Abstract 57-4-278. 
Proof. If $G=F / R$ is nilpotent, say $G_{N+1}=1$, we have $R_{N+1}$ $=F_{N+1}=\left[\left(x_{1}, \cdots, x_{N+1}\right)\right.$, all sets $x_{1}, \cdots, x_{N}$ of generators for $\left.F\right]$. Hence $R=\left[S_{N}, R_{N+1}\right]$ is defined by a finite set of relations.

THEOREM 1.2. In a finitely generated group which is known to be nilpotent ${ }^{5}$ the word-problem is decidable.

Proof. Let $G=F / R$ and $G_{N+1}=1$. Suppose we have an expression for the word $w$ in the form $w=r_{n-1} w_{n}$, where $r_{n-1}$ is in $R$ and $w_{n}$ is in $F_{n}$. By reference to the finitely generated abelian group $F_{n} / F_{n+1}$, we can obtain an expression $w_{n}=r_{n} w_{n+1}, r$ in $R, w_{n+1}$ in $F_{n+1}$, if any such exists. Proceeding thus, either $w=r_{1} r_{2} \cdots r_{N} w_{N+1}$ in $R$, or else, for some $n, w=r_{1} r_{2} \cdots r_{n-1} w_{n}$ where $w_{n}$ is not in $\left[R, F_{n+1}\right]$ and hence $w$ is not in $R$.

A normal subgroup $W$ of the free group $F$ is a word group if it is defined by certain words $w\left(\xi_{1}, \cdots, \xi_{n}\right)$ under all substitutions of elements of $F$ for the $\xi_{i}$. For any group $G$, let $F$ be a denumerably generated free group; the group $W_{G}$ of identical relations for $G$ is the normal subgroup of $F$ defined by all words $w\left(\xi_{1}, \cdots, \xi_{n}\right)$ that equal 1 under all substitutions of elements of $G$ for the $\xi_{i}$.

Lемма 2. Let $F$ be a free group and $W$ a word subgroup of $F$. Then, for each $n \geqq 1, W=\left\{S_{n}, W_{n+1}\right\}$, the word group defined by a finite set $S_{n}$ of words, in at most $n$ indeterminates, together with $W_{n+1}=W \cap F_{n+1}$.

Proof. Induction as for Lemma 1. Consider the set of all relations of the form

$$
r=\prod_{i} c_{i} \cdot s
$$

where the $c_{i}$ are commutators of generators of $F$ of order $n+1$, $\prod c_{i} \neq 1$, and $s$ is in $F_{n+2}$. Each $c_{i}$ contains at most $n+1$ generators. Let $X$ be the set of generators occurring in some $c_{i_{0}}$ in $r$. Substituting $x_{k} \rightarrow 1$ for all generators $x_{i}$ not in $X$, we derive from $r$ a relation

$$
r^{\prime}=\prod^{\prime} c_{i} \cdot s^{\prime}
$$

where $\Pi^{\prime} c_{i}$ is a partial product of that occurring in (1) and contains

s It is understood that $G$ is defined by a finite set of relations, whence a finite set for $F_{n}$ modulo $F_{n+1}$ can be obtained, say, by a simplification of the ReidemeisterSchreier process. It suffices for Theorem 2.1, in fact, to assume that the $G_{n}$ have intersection 1 . To see this, test, for $n=1,2, \cdots$, the two conditions: (i) $w$ is in $\left[R, F_{n}\right]$; and (ii) $w$ is not equal, in $F$, to any product $\prod u_{i} r_{i} u_{i}^{-1}$ where the $u_{i}$ and $r_{i}$ together are of total length less than $n$. For some finite $n$ either (i) must fail and so $w$ is not in $R$, or (ii) must fail, whence $w$ is in $R$. 
at least the factor $c_{i_{0}}$. Therefore, in

$$
r^{\prime \prime}=r \cdot r^{\prime-1}=\prod^{\prime \prime} c_{i} \cdot s^{\prime \prime}
$$

the product contains fewer factors than that in (1). If we repeat this construction, each relation (1) is obtained as a consequence of relations (2), each involving at most $n+1$ generators. Now, all the relations (2) are equivalent, for the purpose of defining $W$, to the corresponding relations $\left(2^{\prime \prime}\right)$ in the generators $x_{1}, \cdots, x_{n+1}$, and, by Lemma 1 , these possess a finite basis $T$ modulo $W_{n+2}$. Thus, if $W$ $=\left\{S_{n}, W_{n+1}\right\}$, then $W=\left\{S_{n}, T, W_{n+2}\right\}$.

THEOREM 2.1. A nilpotent group $G$ possesses a finite basis of identical relations.

Proof. If $G_{N+1}=1$, then $F_{N+1} \subset W_{G} \subset F$. By Lemma 2, $W$ $=\left\{S_{N}, W_{N+1}\right\}$ where $S_{N}$ is finite. But $W_{N+1}=F_{N+1}$ is defined by the single word $\left(\xi_{1}, \cdots, \xi_{N+1}\right)$, whence $W$ has a finite basis. We note that, by multiplying together all the words in this basis, taken with distinct indeterminates, we obtain a single word which constitutes a basis for $W$.

Princeton UnIVersity 